\title{
Occurrence of Aelurostrongylus abstrusus and Troglostrongylus brevior in domestic cats in Greece
}

\author{
Anastasia Diakou ${ }^{1 *}$, Angela Di Cesare ${ }^{2}$, Luciano A. Barros ${ }^{2,3}$, Simone Morelli ${ }^{2}$, Lenaig Halos ${ }^{4}$, Frederic Beugnet ${ }^{4}$ \\ and Donato Traversa ${ }^{2}$
}

\begin{abstract}
Background: Despite the evidence that Mediterranean Europe offers suitable conditions for the biology of felid respiratory metastrongyloids, no updated data on the presence of felid lungworms are available for Greece. Although the cat lungworm Aelurostrongylus abstrusus is considered as enzootic in domestic cats (Felis silvestris catus) living in some areas of continental Greece, conversely, Troglostrongylus brevior, has only been reported in the island of Crete. The present study aimed to evaluate the occurrence of Aelurostrongylus abstrusus and Troglostrongylus brevior in domestic cats from four different Greek locations including islands where European wildcats (Felis silvestris silvestris), believed to be the natural reservoir of T. brevior, are considered absent.

Methods: Faeces were collected from 125 stray cats in the city of Athens, and in Crete, Mykonos and Skopelos Islands, and examined by copromicroscopic techniques for the presence of lungworm larvae. When present, larvae were morphologically and molecularly identified.

Results: The occurrence of A. abstrusus and T. brevior was confirmed in 10 (8\%) and 7 (5.6\%) of the samples, respectively. In particular, T. brevior was detected in domestic cats in the city of Athens, and in Mykonos and Skopelos Islands, where wildcats are not present.

Conclusions: This information illustrates that T. brevior may infect domestic cats regardless of the presence of the natural host. Considering the relevant clinical impact of this nematode especially in young animals, it is advisable to include troglostrongylosis in the differential diagnosis of cat respiratory diseases also where this parasite is unexpected.
\end{abstract}

Keywords: Aelurostrongylus abstrusus, Troglostrongylus brevior, Lungworms, Greece, Greek islands, Domestic cats

\section{Background}

The cat lungworm Aelurostrongylus abstrusus, i.e. the most important respiratory parasite of domestic cats (Felis silvestris catus) worldwide, has been traditionally considered to be the only metastrongyloid affecting $F$. s. catus $[1,2]$. Nonetheless, other respiratory nematodes have recently been recorded from $F$. s. catus either after a long time since the first description, or for the first time. Some of them, e.g. Troglostrongylus subcrenatus

\footnotetext{
* Correspondence: diakou@vet.auth.gr

'Laboratory of Parasitology and Parasitic Diseases, School of Veterinary Medicine, Faculty of Health Sciences, Aristotle University of Thessaloniki, 54124 Thessaloniki, Greece

Full list of author information is available at the end of the article
}

or Oslerus rostratus, still remain of minor and limited relevance under an epizootiological standpoint and are likely to be confined in their wild hosts [1-4]. Conversely, Troglostrongylus brevior has gained high attention in the past few years, for its clinical relevance, especially in young animals and for an apparent expansion in populations of domestic cats $[1,4,5]$.

Aelurostrongylus abstrusus affects domestic cats worldwide, with high prevalence in certain areas [1]. In Greece, the parasite has been found in $1.6 \%$ of the cats in the North of the country [6], and since then it has been sporadically detected in routine parasitological examination of cats (Diakou, unpublished data).

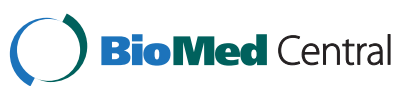

(c) 2015 Diakou et al. Open Access This article is distributed under the terms of the Creative Commons Attribution 4.0 International License (http://creativecommons.org/licenses/by/4.0/), which permits unrestricted use, distribution, and reproduction in any medium, provided you give appropriate credit to the original author(s) and the source, provide a link to the Creative Commons license, and indicate if changes were made. The Creative Commons Public Domain Dedication waiver (http://creativecommons.org/publicdomain/zero/1.0/) applies to the data made available in this article, unless otherwise stated. 
Troglostrongylus brevior was first described in the last century from two species of wild felids in Palestine [7]. It was then found in one European wildcat (Felis silvestris silvestris) from central Italy and in an animal that the author did not clarify whether it was a feral cat or a wildcat [8]. No other records of T. brevior were published in the peer-reviewed literature until 2010, when this lungworm was recorded for the first time in two domestic cats living in Ibiza Island (Spain) [9]. The report from Ibiza brought troglostrongylosis in domestic cats to the fore and since then, this infection has been increasingly recorded in other European islands, i.e. Sicily, Sardinia and Crete $[5,10,11]$, and in areas of continental Italy $[3,4,12-16]$.

In Italy and Greece, T. brevior until now has been described in domestic cats living in areas where the presence of wildcats is documented (Fig. 1) [3-5, 10-17]. A range of reasons indicate that wildcats may be the natural hosts of T. brevior, and that a spill-over of this parasite to domestic cats may occur in certain ecological niches $[1,2,18]$.

Despite the evidence that Mediterranean Europe offers suitable conditions for the biology of felid respiratory metastrongyloids, no updated information is available for the occurrence of $A$. abstrusus in Greece, that has been thus far previously reported only in the northern territories [6]. Additionally, only one record exists for $T$. brevior, i.e. in one kitten from Crete Island [11].

The present article aims to update current knowledge on cat lungworms in Greece, describing the occurrence of $A$. abstrusus and T. brevior in domestic cats from continental and insular regions. Epizootiological and clinical implications of the findings are discussed in relation to different environmental habitats in Greece, both in areas of presence or absence of wildcats.

\section{Methods}

\section{Study design and faeces collection}

In June and July 2015 individual faecal samples of 125 stray cats were collected in four geographic locations in continental and insular Greece, i.e. the city of Athens (23 cats), and the islands of Crete (34 cats), Mykonos (43 cats) and Skopelos (25 cats). These locations were selected based on a study design (see Acknowledgments) including both continental and insular touristic areas of Greece, with varying environments, i.e. dry

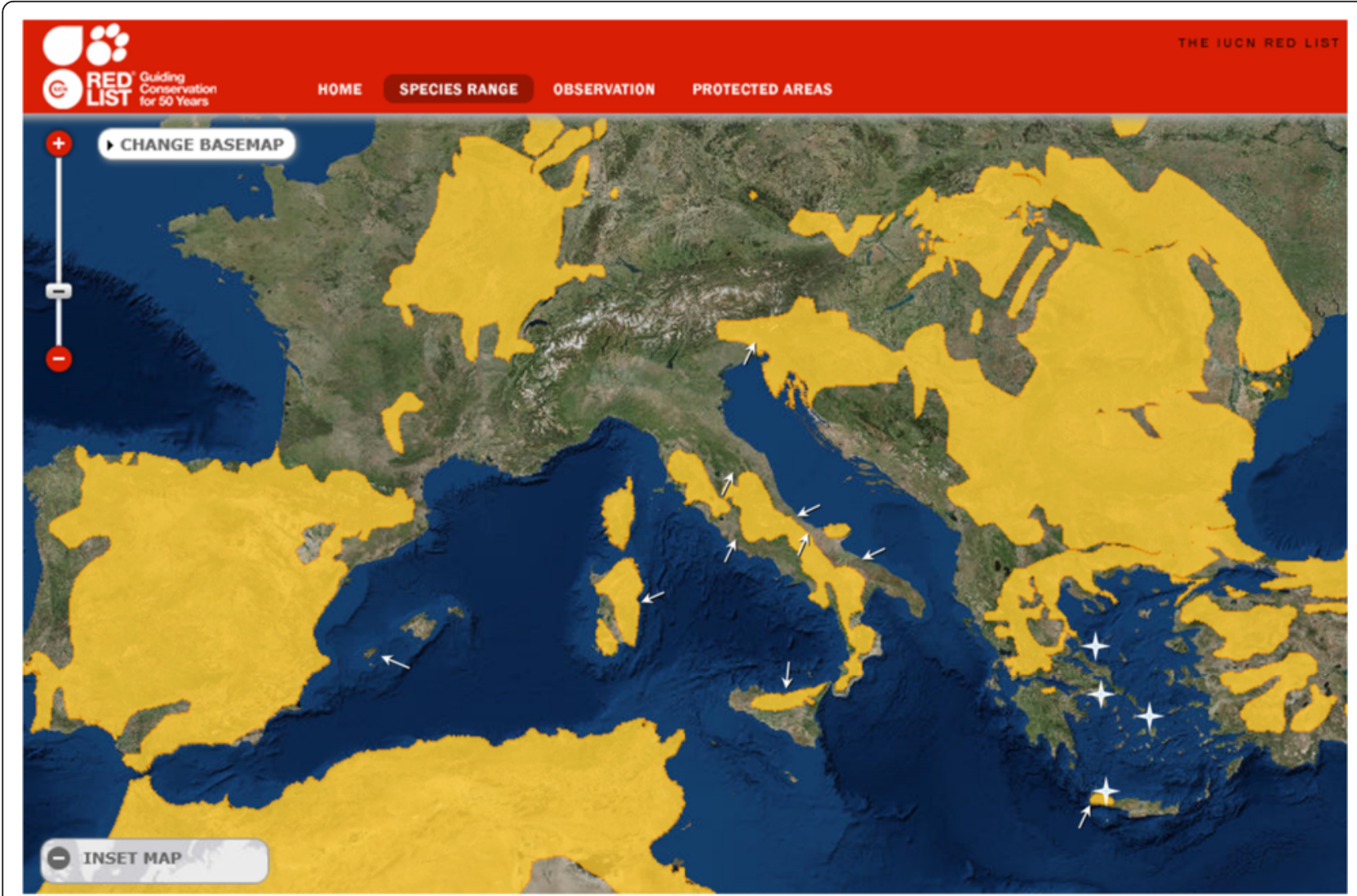

Fig. 1 Map of the range of Felis silvestris silvestris in Southern and Central Europe (modified from the site of the International Union for Conservation of Nature (IUCN, www.iucnredlist.org) Arrows indicate the areas were Troglostrongylus brevior has been documented in domestic cats; asterisks indicate the study areas 
(Crete, Mykonos), forested (Skopelos) and highly urbanised (Athens) areas, and on an axel extending from the south to the north of the country.

Individual faeces were collected from each single cat after a rectal paediatric glycerine enema. All animals were examined with the consent of the local Municipality Authorities.

\section{Parasite detection and identification}

The faecal samples were examined by classical copromicroscopic examination, i.e. $\mathrm{ZnSO}_{4}$ flotation and Baermann method $[19,20]$. The larvae found at the faecal examinations were morphologically studied, measured and identified according to existing morphological descriptions and keys $[1,21,22]$. In addition, DNA was extracted from the purified larvae and molecular identification was conducted using a newly developed molecular assay for the simultaneous detection of A. abstrusus and T. brevior specific DNA [23]. In brief, a multiplex semi-nested PCR using a universal set of primers (i.e. NC1-NC2) in the first step and a combination of forward primers specific for cat respiratory nematodes along with $\mathrm{NC} 2$ primer was used [23].

\section{Results}

At the copromicroscopic examination lungworm larvae were retrieved in the faeces of 15 cats (12\%) living in all examined sites, specifically in 5 (21.7\%), 1 (2.9\%), 7 $(16.3 \%)$ and 2 (8\%) of the cats from Athens, Crete, Mykonos and Skopelos, respectively (Table 1). The identification of the larvae in each positive sample was confirmed both by morphological examination and PCR. Larvae of A. abstrusus (Fig. 2) were found in a total of $10(8 \%)$ cats, i.e. 4 in Athens, 1 in Crete, 3 in Mykonos and 2 in Skopelos, while larvae of T. brevior (Fig. 3) were found in 7 (5.6 \%) cats, i.e. in 1 in Athens, 5 in Mykonos and 1 in Skopelos. Of the 15 positive cats, 13 had a monospecific infection by either $A$. abstrusus (8 animals) or T. brevior (5 animals). Mixed infections (Fig. 4), were detected in two cats, one in Mykonos and one in Skopelos.

Table 1 Prevalence (\% $\% 95 \%$ confidence interval, Cl) of Aelurostrongylus abstrusus (Aab) and Troglostrongylus brevior (Tb) in cats examined from four different areas of Greece

\begin{tabular}{lllll}
\hline Area & No. of cats & Aab $(\% \pm \mathrm{Cl})$ & $\mathrm{Tb}(\% \pm \mathrm{Cl})$ & Total $(\% \pm \mathrm{Cl})$ \\
\hline Athens & 23 & $4(17.4 \pm 7.9)$ & $1(4.3 \pm 4.2)$ & $5(21.7 \pm 17)$ \\
Crete & 34 & $1(2.9 \pm 2.8)$ & 0 & $1(2.9 \% \pm 5.6)$ \\
Mykonos & 43 & $3(7 \pm 3.8)$ & $5(11.6 \pm 4.8)$ & $7^{\mathrm{a}}(16.3 \% \pm 11)$ \\
Skopelos & 25 & $2(8 \pm 5.4)$ & $1(4 \pm 3.9)$ & $2^{\mathrm{a}}(8 \% \pm 10)$ \\
Total & 125 & $10(8 \pm 2.4)$ & $7(5.6 \pm 2)$ & $15^{\mathrm{a}}(12 \% \pm 2.9)$
\end{tabular}

${ }^{a}$ Mixed infections by both parasites were recorded in 2 cats, 1 in Mykonos and 1 in Skopelos

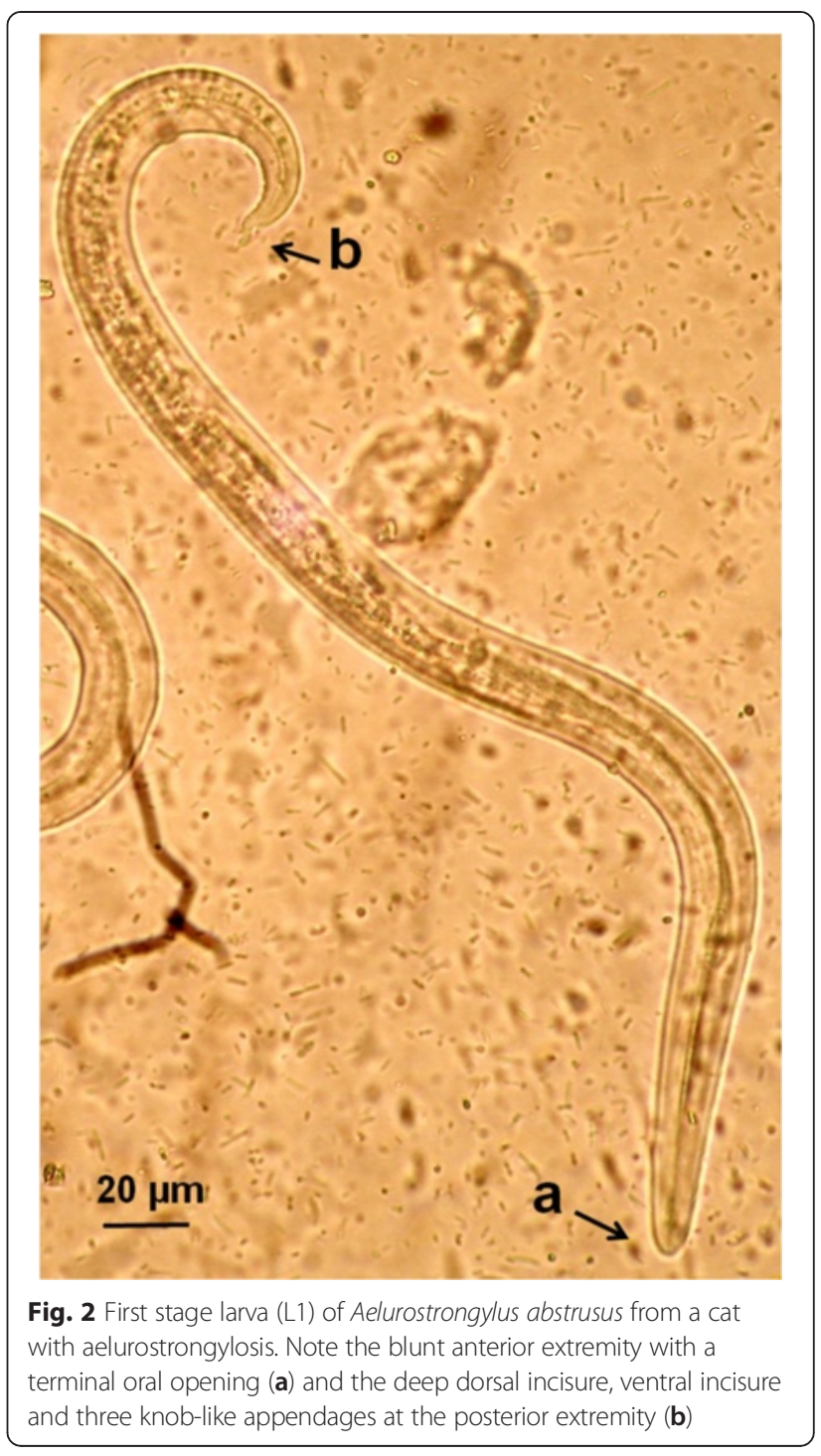

\section{Discussion}

The present data demonstrate that cats living in various Greek islands and in the continental territory of Athens may harbour A. abstrusus and T. brevior, in single or mixed infections, and with high infection rates in some areas (e.g. Athens, Mykonos). Most importantly, this report provides evidence that domestic cats may harbour T. brevior in regions where the natural host $[24,25]$, i.e. the wildcat, is absent. The high level of infection with lungworms in the examined stray cats shows the important role that free-roaming animals may have in the dissemination of these parasites. In fact, the level of veterinary care in stray cats is generally low, thus these animals remain untreated for a long time and disseminate their parasites in the environment. Hence, they act as a source of infection for the intermediate and paratenic hosts, increasing the risk of infection in other susceptible hosts living in the same regions. 


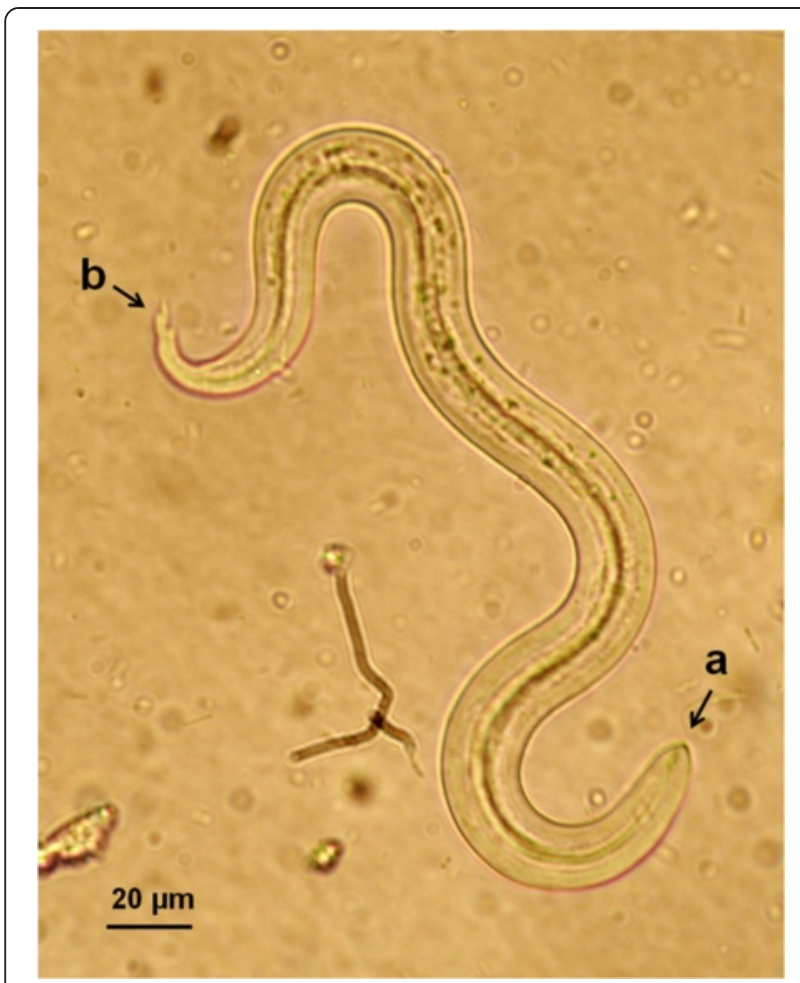

Fig. 3 First stage larva (L1) of Troglostrongylus brevior from a cat with troglostrongylosis. Note the pointed anterior extremity with a subterminal oral opening (a) and the dorsal and ventral incisure at the posterior extremity (b)
The present study adds new knowledge on the geographical distribution of the cat lungworm $A$. abstrusus in Mediterranean Europe, as no updated and multi-site information were available for Greece and no data were obtained for the study areas thus far. It is here shown that cat aelurostrongylosis may occur with relatively high infection rates in some parts of Greece (Table 1), i.e. higher than the values previously recorded in the north of the country [6]. The only information available for $T$. brevior in Greece is a single case report from Crete [11]. This current study shows that this nematode can infect free-roaming domestic cats in continental Greece and islands other than Crete, in a range of latitudes extending from $35^{\circ} 51^{\prime} \mathrm{N}$ (Crete) to $39^{\circ} 12^{\prime} \mathrm{N}$ (Skopelos) and longitudes from $23^{\circ} 72^{\prime} \mathrm{E}$ (Athens) to $25^{\circ} 35^{\prime} \mathrm{E}$ (Mykonos). Interestingly, both $A$. abstrusus and T. brevior were found in varying environments, e.g. dry (Mykonos), forested (Skopelos) and highly urbanised (Athens) areas. It is important to note that the dry climate and poor vegetation characterising Mykonos and the urban environment of Athens do not represent an obstacle to the life-cycle of these mollusctransmitted nematodes. On the contrary, the highest presence of both lungworms was recorded in these two study areas, thus indicating that the life-cycle of feline lungworms can be completed, as long as even limited habitats ensure the required conditions for the larval and intermediate host survival.

These results demonstrate that $T$. brevior is present in more geographical areas than previously known and that,

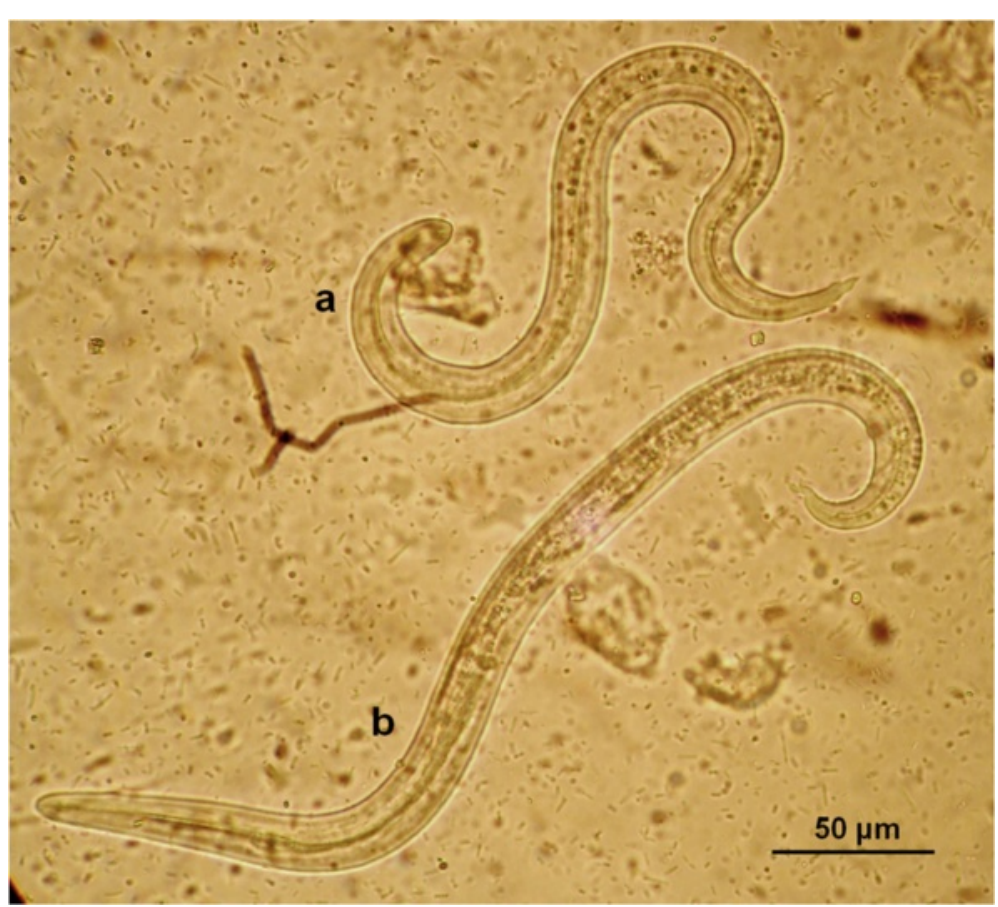

Fig. 4 First stage larvae (L1) of Troglostrongylus brevior a and Aelurostrongylus abstrusus $\mathbf{b}$ from a cat with a mixed infection 
in given regions, domestic cats harbour T. brevior regardless of the occurrence of wild reservoirs. At the moment it is still not ultimately established: (i) to what extent T. brevior occurs in domestic cats living in countries other than Italy, which is the region where this nematode has been recorded more frequently thus far [26]; (ii) if the reports of the last few years are due to a possible affiliation of $T$. brevior in domestic felids mostly in confined areas; and/or (iii) whether some epizootiological factors enforce infections of domestic cats with lungworms of wildlife.

The hypothesis that some drivers may have recently promoted an overflow of $T$. brevior from wildlife to domestic cats is realistic, based on different epizootiological, clinical and biological evidence [1, 4, 26]. As a key example, in a recent survey, only one cat of about 200 examined animals in northern Italy scored positive for T. brevior. This cat lived in the easternmost area of the only region of northern Italy where $F$. s. silvestris is present [4]. At the same time, cats examined in another northern territory of Italy where wildcats are absent, were highly infected with $A$. abstrusus but $T$. brevior was not found [4]. The same study showed that, in some mountainous and hilly areas of central Italy, aelurostrongylosis and troglostrongylosis have a similar distribution [4] despite the fact that T. brevior in domestic cats of the same regions was negligible until about ten years ago [3].

It is likely that under particular conditions (e.g. presence of infected wildcats facilitating the spread of infection, boundaries of islands or mountainous regions) $T$. brevior can change its usual affiliation and infect domestic cats, as in the case in Italy and Crete $[1,11,26$, 27]. It is difficult to draw a definitive explanation on the occurrence of cat troglostrongylosis in regions where the natural host responsible for spread of infection (i.e. $F$. s. silvestris) is not documented, i.e. Ibiza Island [9] and the Greek areas herein examined. The presence of wildcats in the Balearic Islands is uncertain and hypothesized only in the island of Mallorca [17, 28, 29]. Similarly, in Greece, populations of $F$. s. silvestris live only in forested areas and wetlands of continental regions [17], and in the Chania region of Crete Island (Fig. 1), i.e. where the first case of troglostrongylosis in a domestic cat from Greece has been recently described [11]. It is possible that, in given regions, this parasite has adapted to domestic cats in prehistorical times, before the present coastal arrangement appeared (c. 7000 BC - 4000 BC) [30], when the wildcat or a common ancestor was likely present. In such conditions, the nematode might have remained confined in domestic cats in geographical segregation, such as small islands (e.g. Ibiza, Mykonos), thus explaining why it has remained practically unknown until the recent descriptions. It is also possible that cats travelling with their owners in recent years from endemic areas like Italy, to popular and touristic spots could have imported this lungworm, or that movements of paratenic hosts (migratory birds, rodents moved with international trade of goods in ships, etc.) may have introduced infective stages of $T$. brevior. The absence of $T$. brevior in cats examined in Crete, despite the fact that this parasite is present on the island [11], could be due to a range of reasons. Indeed, $T$. brevior is in general less common than A. abstrusus, thus its absence in 34 examined cats is not surprising. The number of sampled animals could have reduced the chance of detection if this nematode has a limited distribution in local domestic cats and is still confined in wildlife. Moreover, most examined cats were from a different area (Stavros) from where the first case of troglostrongylosis was found in 2014 (city of Chania). It is important to note that $A$. abstrusus has been found here in only one cat, thus suggesting that lungworms in Crete may have a limited distribution compared to other areas.

Further studies could assist in exploring the genetic structure of $T$. brevior populations affecting both wild and domestic hosts from different geographic areas. These investigations could be important to understand if certain subpopulations of lungworms can selectively infect each different felid species in all enzootic areas or in given regions, or to the contrary, F. s. catus and $F$. s. silvestris share the same genetic populations of lungworms in all these areas. In fact, subpopulations of other strongylid species, i.e. hookworms and cyathostomins, were previously indicated to selectively infect different animal species [31, 32].

Finally, as a practical output, it is advisable that troglostrongylosis is definitively included in the differential diagnosis of respiratory diseases of cats, also in regions where T. brevior is unexpected. In fact, in the near future we could be faced with the spread of $T$. brevior in domestic cats, both where the natural hosts are expanding, i.e. central Italy [3, 4, 26, 27], and where wildcats are absent. This is of pivotal importance because a timely detection of $T$. brevior implies a prompt anthelmintic treatment with spot-on molecules that recently proved efficacious in treating cat troglostrongylosis in natural and/or experimental conditions, e.g. emodepside [14], eprinomectin [33, 34] and moxidectin [35].

\section{Competing interests}

The present findings were obtained during a survey carried out to investigate the occurrence of zoonotic intestinal helminths and vector-borne pathogens in stray cats from touristic areas of Greece and supported by Merial, of which LH and FB are employees. ADC holds a post-doctoral fellowship on parasites other than cat lungworms and has participated in the present study in the framework of her freedom of scientific research.

\section{Authors' contributions}

$A D, A D C$ and DT participated in project activities, drafted the manuscript and revised it critically. $L A B$ and SM participated in the field and laboratory work. 
$\mathrm{LH}$ and FB participated in drafting the manuscript for intellectual content. All authors read and approved the final manuscript.

\section{Acknowledgements}

The authors would like to acknowledge the veterinarians Tania Aeriniotaki, Athina Tsokana, Dimitris Papaioannou, Niki Lemoni, Christos Ktenas and the biologist Despina Migli for their valuable assistance. The authors are also grateful to Paolo Matteo Accettura and Francesca Laiacona for their support in the laboratory activities.

\section{Author details}

'Laboratory of Parasitology and Parasitic Diseases, School of Veterinary Medicine, Faculty of Health Sciences, Aristotle University of Thessaloniki, 54124 Thessaloniki, Greece. ${ }^{2}$ Faculty of Veterinary Medicine, Teaching Veterinary Hospital, Località Piano d'Accio snc, 64100 Teramo, Italy. ${ }^{3}$ Faculdade de Medicina Veterinaria MSV/UFF, Rua Vital Brasil Filho, 64 Icaraí, Niterói, Rio de Janeiro 24230-340, Brazil. ${ }^{4}$ Merial S.A.S, 29 Av Tony Garnier, 69007 Lyon, France.

\section{Received: 5 October 2015 Accepted: 6 November 2015}

\section{Published online: 14 November 2015}

\section{References}

1. Traversa D, Di Cesare A. Feline lungworms: what a dilemma. Trends Parasitol. 2013;2:423-30.

2. Traversa D, Di Cesare A. Cardio-pulmonary parasitic nematodes affecting cats in Europe: unraveling the past, depicting the present, and predicting the future. Frontiers Vet Sci. 2014; doi: 10.3389/fvets.2014.00011

3. Di Cesare A, Di Francesco G, Frangipane Di Regalbono A, Eleni C, De Liberato C, Marruchella G, et al. Retrospective study on the occurrence of the feline lungworms Aelurostrongylus abstrusus and Troglostrongylus spp. in endemic areas of Italy. Vet J. 2015;203:233-8.

4. Di Cesare A, Veronesi F, Grillotti E, Manzocchi S:, Perrucci S, Beraldo P, et al. Respiratory nematodes in cat populations of Italy. Parasitol Res. 2015; 114(12):4463-9. in press.

5. Brianti E, Gaglio G, Giannetto S, Annoscia G, Latrofa MS, Dantas-Torres F, et al. Troglostrongylus brevior and Troglostrongylus subcrenatus (Strongylida: Crenosomatidae) as agents of broncho-pulmonary infestation in domestic cats. Parasit Vectors. 2012;5:178.

6. Haralabidis TS. Contribution in the study of cat parasites and their significance in Public Health. PhD Thesis. Scientific Yearbook of The Faculty of Veterinary Medicine, Aristotle University of Thessaloniki, Greece 1978;18: 237-379 (In Greek with English abstract)

7. Gerichter CB. Studies on the nematodes parasitic in the lungs of Felidae in Palestine. Parasitology. 1949;39:251-62.

8. Paggi L. Segnalazione, in Italia Centrale, di Troglostrongylus sp. parassita dei polmoni di felidi. Parassitologia. 1959;1:80-1 (In Italian).

9. Jefferies R, Vrhovec MG, Wallner N, Catalan DR. Aelurostrongylus abstrusus and Troglostrongylus sp. (Nematoda: Metastrongyloidea) infections in cats inhabiting Ibiza, Spain. Vet Parasitol. 2010;173:344-8.

10. Tamponi C, Varcasia A, Brianti E, Pipia AP, Frau V, Pinna Parpaglia ML, et al. New insights on metastrongyloid lungworms infecting cats of Sardinia. Italy Vet Parasitol. 2014;203:222-6.

11. Diakou A, Di Cesare A, Aeriniotaki T, Traversa D. First report of Troglostrongylus brevior in a kitten in Greece. Parasitol Res. 2014;113:3895-8.

12. Brianti E, Gaglio G, Napoli E, Falsone L, Giannetto S, Latrofa MS, et al. Evidence for direct transmission of the cat lungworm Troglostrongylus brevior (Strongylida: Crenosomatidae). Parasitology. 2013;140:821-4.

13. Di Cesare A, Frangipane Di Regalbono A, Tessarin C, Seghetti M, lorio R, Simonato G, et al. Mixed infection by Aelurostrongylus abstrusus and Troglostrongylus brevior in kittens from the same litter in Italy. Parasitol Res. 2014;113:613-8

14. Di Cesare A, lorio R, Crisi P, Paoletti B, Di Costanzo R, Dimitri CF, et al. Treatment of Troglostrongylus brevior (Metastrongyloidea, Crenosomatidae) in mixed lungworm infections using spot-on emodepside. J Feline Med Surg. 2015;17:181-5.

15. Traversa D, Romanucci M, Di Cesare A, Malatesta D, Cassini R, lorio R, et al. Gross and histopathological changes associated with Aelurostrongylus abstrusus and Troglostrongylus brevior in a kitten. Vet Parasitol. 2014;201:158-62.

16. Traversa D, Lepri E, Veronesi F, Paoletti B, Simonato G, Diaferia M, et al. Metastrongyloid infection by Aelurostrongylus abstrusus, Troglostrongylus brevior and Angiostrongylus chabaudi in a domestic cat. Int J Parasitol. 2015. doi:10.1016/j.ijpara.2015.05.005.

17. Yamaguchi N, Kitchener A, Driscoll C, Nussberger B. Felis silvestris. The IUCN Red List of Threatened Species. Version 2015.2. <www.iucnredlist.org>. Accessed 25 August 2015.

18. Traversa D. Response to Otranto et al.: Lungworms in domestic and wild felids: dilemmas still persisting. Trends Parasitol. 2014;30:53-4

19. MAAF. Manual of Veterinary Parasitological Laboratory Techniques, Ministry of Agriculture, Fisheries and Food (MAFF). London: Her Majesty's Stationary Office: 1986.

20. Thienpont D, Rochette F, Vanparijs OFJ. Diagnosing helminthiasis by coprological examination. 2nd ed. Beerse (Belgium): Janssen Research Foundation; 1986.

21. Sloss MW, Kemp RL, Zajac AM. Faecal examination: dogs and cats. In: Veterinary Clinical Parasitology. 6th ed. AMES: lowa State University Press; 1994.

22. Brianti E, Giannetto S, Dantas-Torres F, Otranto D. Lungworms of the genus Troglostrongylus (Strongylida: Crenosomatidae): neglected parasites for domestic cats. Vet Parasitol. 2014;202:104-12.

23. Di Cesare A, Veronesi F, Frangipane Di Regalbono A, lorio R, Traversa D. Novel molecular assay for the simultaneous identification of neglected lungworms and heartworms affecting cats. J Clin Microbiol. 2015;53(9): 3009-13. JCM.00901-15.

24. Falsone L, Brianti E, Gaglio G, Napoli E, Anile S, Mallia E, et al. The European wildcats (Felis silvestris silvestris) as reservoir hosts of Troglostrongylus brevior (Strongylida: Crenosomatidae) lungworms. Vet Parasitol. 2014;205:193-8.

25. Otranto D, Cantacessi C, Dantas-Torres F, Brianti E, Pfeffer M, Genchi C, Guberti V, Capelli G, Deplazes P. The role of wild canids and felids in spreading parasites to dogs and cats in Europe. Part II: Helminths and arthropods. Vet Parasitol. 2015; doi: 10.1016/j.vetpar.2015.04.020

26. Di Cesare A, Veronesi F, Traversa D. Felid lungworms and heartworms in Italy: more questions than answers? Trends Parasitol. 2015 in press.

27. Veronesi F., Traversa D., Lepri E., Morganti G., Vercillo F., Grelli D., Cassini R., Marangi M., lorio R., Ragni B., Di Cesare A. Occurrence of lungworms in European wildcats (Felis silvestris silvestris) of central Italy. JWD. 2015 in press.

28. Groves CP. Feral mammals of the Mediterranean islands: documents of early domestication. In: Clutton-Brock J, editor. The Walking Larder: Patterns of Domestication, Pastoralism, and Predation. Oxon: Routledge; 2015. p. 46-57.

29. Garcia-Perea R. Felis silvestris Schreber, 1777. In: Palomo L, Gisbert J, Blanco JC, editors. Atlas y Libro Rojo de los Mamíferos Terrestres de España. Madrid: Dirección General para la Biodiversidad -SECEM-SECEMU; 2007. p. 333-8.

30. van Andel TH, Shackleton JC. Late Paleolithic and Mesolithic Coastlines of Greece and the Aegean. J Field Archaeol. 1982;9:445-54.

31. Hu M, Chilton NB, Zhu X, Gasser RB. Single-strand conformation polymorphism-based analysis of mitochondrial cytochrome c oxidase subunit 1 reveals significant substructuring in hookworm populations. Electrophoresis. 2002;23:27-34.

32. Traversa D, Kuzmina T, Kharchenko VA, lorio R, Klei TR, Otranto D. Haplotypic variability within the mitochondrial gene encoding for the cytochrome $\mathrm{C}$ oxidase 1 (cox1) of Cylicocyclus nassatus (Nematoda, Strongylida): evidence for an affiliation between parasitic populations and domestic and wild equid hosts. Vet Parasitol. 2008;156:241-7.

33. Giannelli A, Brianti E, Varcasia A, Colella V, Tamponi C, Di Paola G, et al. Efficacy of Broadline ${ }^{\circledast}$ spot-on against Aelurostrongylus abstrusus and Troglostrongylus brevior lungworms in naturally infected cats from Italy. Vet Parasitol. 2015;209:273-7.

34. Knaus M, Visser M, Chester TS, Rehbein S. Efficacy of Broadline ${ }^{\circledast}$ (Merial) against induced infection with Troglostrongylus brevior (Nematoda, Metastrongyloidea). Merial Abstract and Poster Book "New trends in the control of parasites in dogs and cats", WAAVP, Liverpool, 16-20 August 2015, p. 87-89.

35. Crisi PE, Traversa D, Di Cesare A, Luciani A, Civitella C, Santori D, Boari A. Irreversible pulmonary hypertension associated with Troglostrongylus brevior infection in a kitten. Res Vet Sci, in press, 2015; doi: 10.1016/j.rvsc.2015.08.019. 